\title{
Malignant peritoneal mesothelioma with possible hormone association and multiple endocrine neoplasia in a young female with an indolent clinical course
}

\author{
Juliana M Kling1, Paru David', J. Scott Kriegshauser², Henry D. Tazelaar ${ }^{3}$, Helen Ross ${ }^{4}$ \\ 1. Division of Women's Health in the Department of Internal Medicine, Mayo Clinic, Scottsdale, USA. 2. Department of \\ Radiology, Mayo Clinic, Scottsdale, USA. 3. Department of Laboratory Medicine and Pathology, Mayo Clinic, Scottsdale, \\ USA. 4. Division of Oncology in the Department of Internal Medicine, Mayo Clinic, Scottsdale, USA.
}

Correspondence: Juliana M Kling. Address: 13737 North 92nd Street, Scottsdale, AZ 85260, USA. E-mail: kling.juliana@mayo.edu

Received: July 25, 2014

Accepted: October 11, 2014

Online Published: October 27, 2014

DOI : $10.5430 /$ crcp.v2n1p75

URL: http://dx.doi.org/10.5430/crcp.v2n1p75

\begin{abstract}
Malignant peritoneal mesotheliomas are rare, aggressive tumors that carry an overall 5\% 5-year survival rate. This case highlights a young woman with an incidentally discovered malignant peritoneal mesothelioma which grew during pregnancy and has had an indolent course despite the inherent aggressive nature and pathologic appearance of this cancer. Immunohistochemical staining did not show hormone receptors however clinically it is believed she had hormone associated disease. Follow up over the last five years has demonstrated waxing and waning abdominal CT findings that resolve with menses and have not been amenable to biopsy and the patient has remained well. Although prognosis is considered poor for malignant peritoneal mesothelioma, factors such as gender and hormone association may lead to a more indolent course. This patient has also been diagnosed with multiple endocrine neoplasia (MEN1) after being found to have primary hyperparathyroidism and an islet cell tumor. This is only the second reported case of MEN1 and malignant peritoneal mesothelioma.
\end{abstract}

\section{Key words}

Malignant peritoneal mesothelioma, Multiple endocrine neoplasia, Hormone associated mesothelioma

\section{I ntroduction}

Mesotheliomas are aggressive neoplasms that arise from mesothelial cells in the serous lining of the pleura, peritoneum, pericardium and tunica vaginalis testis with the pleural site being most common ${ }^{[1]}$. Peritoneal mesotheliomas account for only $30 \%$ of diagnoses ${ }^{[2]}$. Like pleural mesothelioma, the pathogenesis in many patients is linked to asbestos exposure by ingestion or through lymphatic spread of fibers ${ }^{[3,4]}$. We present a case of an incidentally discovered malignant peritoneal mesothelioma which grew during pregnancy and resolved following delivery. Despite negative hormone receptors, it appears likely based on her clinical course that she had hormone associated disease. Malignant peritoneal mesothelioma has a poor prognosis with an overall 5\% five-year survival ${ }^{[2]}$. Female gender and age younger than 40 years correlates with better prognosis ${ }^{[5]}$. Our patient remains clinically disease free for greater than five years. Her case is unusual in that 
her pathology is consistent with aggressive disease while her clinical course has been indolent and in that she has other diagnoses consistent with multiple endocrine neoplasia (MEN1).

\section{Case presentation}

A 25-year-old female presented for evaluation of right lower quadrant abdominal pain and was found to have a pancreatic mass. Endoscopic ultrasound demonstrated a pancreatic body mass and biopsy confirmed an islet cell tumor that was found to be non-functioning. Planned surgical resection was postponed secondary to pregnancy. After a successful delivery, a repeat CT abdomen and pelvis showed enlargement of the mass as well as possible metastases to the omentum (see Figure 1).
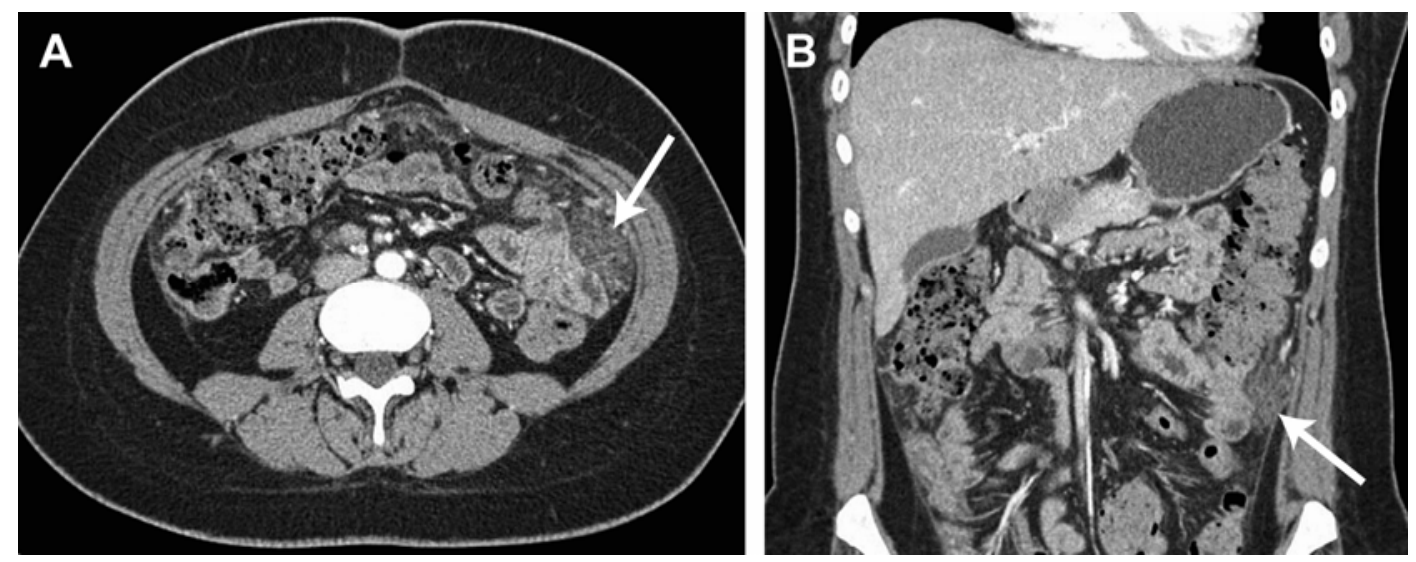

Figure 1. CT abdomen and pelvis. A. Axial image with arrow showing omental mass. B. Coronal image with arrow showing omental mass.

Figure 2. Omental epithelial malignant mesothelioma. Tumor forms two small nodules on the omental surface. (subset) Higher power shows the presence of epithelioid cells with moderate cytologic atypia infiltrating a desmoplastic stroma.

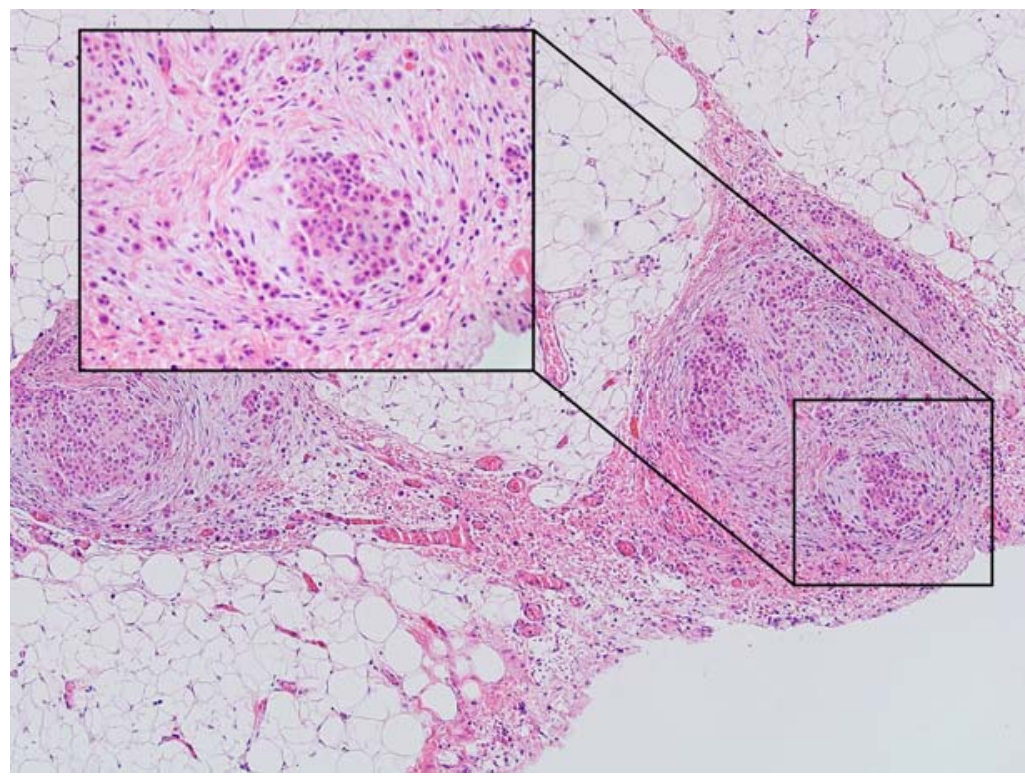

At laparoscopy the distal pancreatic mass was resected and multiple omental biopsies were taken. The omentum and mesentery appeared normal grossly through the laparoscope except for some evidence of prior inflammation in the form of filmy adhesions to the abdominal wall. Pathology showed a $3.0 \mathrm{~cm}$ completely excised well differentiated endocrine tumor of the pancreas with vascular invasion but no perineural invasion and $<2$ mitoses per HPF. The grossly normal omentum showed multiple microscopic foci of diffuse epithelial malignant mesothelioma (see Figure 2). Tumor cells were set in a myxoid stroma. They were arranged in small clusters and linear arrays. Tumor cells expressed CK 5/6, WT-1, keratin 
AE1/AE3 and calretinin but failed to express estrogen or progesterone receptor protein (see Figure 3). The diagnosis of mesothelioma was confirmed by three expert pathologists at our institution, two of whom are members of the United States and Canadian Mesothelioma Panel. An external confirmatory review was provided by an outside pathologist, also a member of the Panel, who is considered a worldwide authority on mesothelioma.
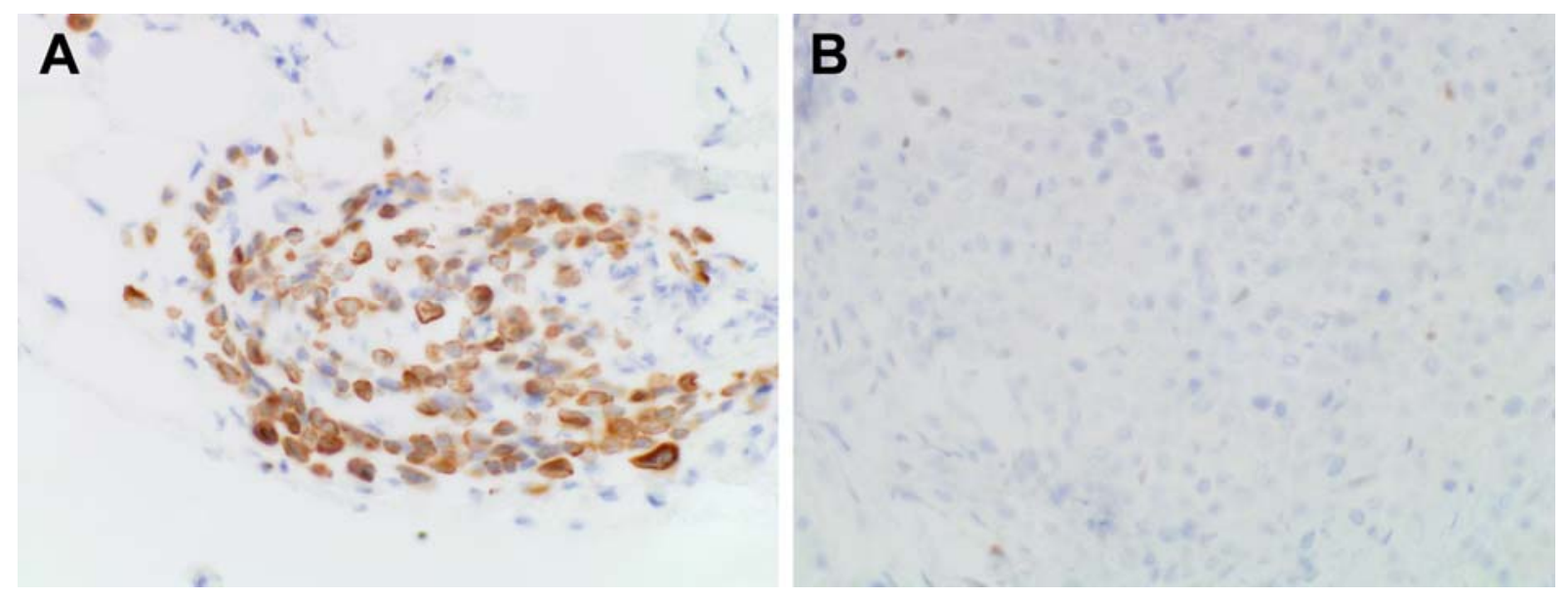

Figure 3. Omental malignant mesothelioma. A. The tumor cells are reactive with antibodies to cytokeratin 5/6. B. The tumor cells fail to react with antibodies to estrogen receptor protein.

The patient was seen in consultation by medical oncology and had no evidence of residual or recurrent mesothelioma by PET CT. She was given a clinical diagnosis of mesothelioma associated with pregnancy that was felt to be functionally hormone associated based on her clinical course despite negative staining for hormone receptors. She denied any risk factors for asbestos exposure and no asbestos fibers were visible in the pathology specimen. In the absence of measurable disease, she was not treated with chemotherapy. Subsequent nodules identified five months later on her hand and back have proven to be lipomas. She had resection of an angiofibroma from her face one year after diagnosis. Pathology showed a fibrous papule. She has had occasional recurrences of the abdominal pain that was her presenting symptom years ago. Repeat PET/CT and CT scans over five years have shown waxing and waning mesenteric and omental stranding raising concern for recurrence but no clear nodules or masses have been seen. Thus far the imaging abnormalities always resolve after her menses making repeat biopsy impossible. The patient avoids exogenous hormones and further pregnancy.

Primary hyperparathyroidism was diagnosed incidentally three years after her mesothelioma diagnosis in response to elevated serum calcium during routine follow up. She was given a diagnosis of MEN1, having had two of the three characteristic tumors including parathyroid, endocrine-gastrointestinal tract or pancreas and pituitary ${ }^{[6]}$. Lipomas can be seen in as many as twenty to thirty percent of MEN1 patients ${ }^{[7]}$. A brain MRI did not demonstrate any pituitary abnormalities. Initial parathyroid surgery demonstrated an adenoma but not the three gland hyperplasia that is characteristic for MEN1. However, she developed a recurrence of her hyperparathyroidism one year later and underwent a subtotal parathyroidectomy for parathyroid hyperplasia. She also developed a right renal pelvis stone requiring lithotripsy. She has no known family history of MEN, and thus does not meet diagnostic criteria for familial MEN1. Her endocrinologist believes that she may be the index member of an undiagnosed kindred with MEN1. Ongoing follow up will include MRI and serum calcium.

\section{Discussion}

In the U.S., 200-400 cases of peritoneal mesothelioma are diagnosed yearly accounting for 10\%-30\% of all mesothelioma cases ${ }^{[2]}$. Five subtypes of mesothelial proliferation occur in the peritoneum: diffuse malignant (histologically similar to pleural mesothelioma), deciduoid (a variant of epithelial diffuse mesothelioma), well-differentiated papillary, multicystic 
(cystic), and adenomatoid tumor (a benign mesothelial tumor) ${ }^{[8]}$. Of the subtypes, the diffuse malignant and deciduoid variants confer the worst prognosis. Well-differentiated mesothelial proliferation predominantly affects the pelvic peritoneum of women without asbestos exposure ${ }^{[8]}$. This form demonstrates low metastatic potential and an excellent prognosis. Deciduoid peritoneal mesothelioma has been described in patients during and after pregnancy and hormone changes may contribute to its development ${ }^{[1]}$.

Peritoneal mesothelioma is rare and can be difficult to diagnose due to non-specific presenting symptoms and the broad differential which includes ovarian and colon cancer ${ }^{[1,3,8]}$. CT scans of the abdomen and pelvis, as well as PET/CT scans, can be useful in diagnosis and follow up ${ }^{[3]}$. Most tumors are epithelioid with a tubulopapillary histologic pattern ${ }^{[8]}$. Differentiation from adenocarcinoma can be difficult histologically and, therefore, pathologic evaluation requires immunohistochemistry using a panel of antibodies ${ }^{[9]}$. Markers such as cytokeratin 5/6 and CEA can be helpful in distinguishing mesothelioma from carcinoma. Hormone receptor staining, including estrogen and progesterone, may be useful in distinguishing mesothelioma from serous tumors of the ovary and peritoneum although mesotheliomas can express hormone receptors ${ }^{[9-11]}$.

Treatment of mesothelioma may include chemotherapy, palliative or definitive surgery and occasionally abdominal radiation ${ }^{[3]}$. Cytoreductive surgery (CRS) and perioperative intraperitoneal chemotherapy (PIC), including hyperthermic intraperitoneal chemotherapy (HIPEC), have been utilized in selected patients with 5-year survivals $29 \%$-63\% reported in nonrandomized trials ${ }^{[2,3]}$.

Unlike pleural mesothelioma, a substantial cohort of women with diffuse peritoneal epithelial mesotheliomas may have a more indolent course and tumor morphology may not reliably predict tumor behavior ${ }^{[12]}$. In 2001 Kerrigan et al. compared tumor morphology in twenty five women with diffuse malignant mesothelioma with either an indolent course ( $>$ four year survival) or an aggressive course ( $<$ four year survival). They found no reliable morphological features that identified favorable and unfavorable tumors. Because of the uncertain behavior based on histopathology, their conclusion was that all women with diffuse malignant mesothelioma should be treated aggressively. Based on the observation that morphology may not predict behavior, it seems equally reasonable to pursue a course of close vigilance and to reserve aggressive therapy for patients with signs of progression.

Our patient's pathology was consistent with the diffuse malignant form of mesothelioma, while her clinical history and course mirrors that of a well-differentiated papillary or deciduoid subtype. Based on her pathology, she could have been a candidate for aggressive therapy after which the medical team would no doubt have congratulated itself on a successful outcome. However, remarkably, her tumor regressed on its own as assessed at laparoscopy and she remains clinically without evidence of progression of disease by CT and PET imaging. The reasons for the discordance between the histologic appearance and clinical course are not clearly known. It seems likely that the hormonal changes associated with pregnancy and delivery may have been a contributor and it has been recommended that she avoid exogenous hormone exposure. The view that this cancer may be hormone sensitive is supported by CT findings which wax and wane during her menstrual cycle, with resolution of CT abnormalities after menses. It has been decided jointly by the patient and the care team that the harms of intraperitoneal chemotherapy could outweigh potential benefit in the absence of evidence of recurrence and she continues close symptomatic and radiographic follow up. It is intended that aggressive therapy would be reconsidered at the earliest sign of active mesothelioma.

During the course of follow up, our patient was diagnosed with two additional endocrine tumors. A case report by Nishimura et al. described a young female patient with MEN1 who was found to have an incidental omental mesothelioma during abdominal surgery ${ }^{[6]}$. The authors proposed a genetic or environmental link or genetic instability that led to both conditions. There is no established association between endocrine neoplasia and mesothelioma, although it is interesting that this is the second reported patient with this association. 


\section{Conclusion}

Peritoneal mesothelioma is a rare and typically aggressive tumor that accounts for approximately $10 \%-30 \%$ of all mesotheliomas. Our patient presented with a histologically aggressive, malignant form of mesothelioma which has had a surprisingly indolent clinical course and is potentially associated with MEN1. Based on her clinical course, it appears that her mesothelioma is hormone associated despite negative estrogen receptor staining and this association may contribute to her long disease free interval without specific therapy and her overall indolent course.

\section{References}

[1] Bridda A, Padoan I, Mencarelli R. Peritoneal Mesothelioma. A Review. Med Gen Med. 2007; 9(2): 32. PMid: 17955087.

[2] Baratti D, Kusamura S, Deraco M. Diffuse Malignant Peritoneal Mesothelioma. Systematic Review of Clinical Management and Biological Research. Journal of Surgical Oncology. 2011; 103: 833-831. http://dx.doi.org/ 10.1002/jso.21787

[3] Mirarabshahii P, Pillai K, Chua TC, Pourghomali MH, Morris, DL. Diffuse malignant peritoneal mesothelioma - An update on treatment. Cancer Treat Rev. 2012; 38(6): 605-12. http://dx.doi.org/ DOI: 10.1016/j.ctrv.2011.10.006

[4] Boffetta P. Epidemiology of peritoneal mesothelioma: a review. Annals of Oncology. 2006; 18: 985-990. http://dx.doi.org/ 10.1093/annonc/mdl345

[5] Cao C, Yan TD, Deraco M, Elias D, Glehen O, Levine EA, et al. Sugarbaker PH; Peritoneal Surface Malignancy Group: Importance of gender in diffuse malignant peritoneal mesothelioma. Annals of Oncology. 2012; 23(6): 1494-8. http://dx.doi.org/ 10.1093/annonc/mdr477

[6] Nishimura Y, Yamashita K, Yumita W, Yamazaki M, Katai M, Sakurai A, et al. Multiple Endocrine Neoplasia Type 1 with Unusual Concomitance of Various Neoplastic Disorders. Endocrine Journal. 2004; 51(1): 75-81. http://dx.doi.org/ 10.1507/endocrj.51.75

[7] Marini F, Falchetti A, Del Monte F, Sala SC, Gozzini A, Luzi E, et al. Multiple endocrine neoplasia type 1. Orphanet Journal of Rare Diseases. 2006; 38: 1-9. http://dx.doi.org/ 10.1186/1750-1172-1-38

[8] Hassan R, Alexander R. Nonpleural Mesotheliomas: Mesothelioma of the Peritoneum, Tunica Vaginalis, and Pericardium. Hematol Oncol Clin N Am. 2005; 1067-1087. http://dx.doi.org/ 10.1016/j.hoc.2005.09.005

[9] Husain AN, Colby TV, Ordonez NG, Krausz T, Borczuk A, Cagle PT, et al. Guidelines for Pathologic Diagnosis of Malignant Mesothelioma. Arch Pathol Lab Med. 2009; 133: 1317-1331. PMid: 19653732.

[10] Pinton G, Brunelli E, Murer B, Puntoni R, Puntoni M, Fennell DA, et al. Estrogen receptor-beta affects the prognosis of human malignant mesothelioma. Cancer Res. 2009; 69(11): 4598-604. http://dx.doi.org/ 10.1158/0008-5472.CAN-08-4523

[11] Jennings CJ, O’Grady A, Cummins R, Murer B, Kay EW, Harvey BJ, et al. Steroid Hormone Receptor Expression in Malignant Pleural Mesothelioma: Estrogen Receptor Beta Isoforms Have Prognostic Value. Am J Respir Crit Care Med. 2012; 185 : A5337.

[12] Kerrigan SA, Turnnir RT, Clement PB, Young RH, Churg A. Diffuse malignant epithelial mesotheliomas of the peritoneum in women: a clinicopathologic study of 25 patients. Cancer. 2002; 94(2): 378-85. http://dx.doi.org/ 10.1002/cncr.10209 